\title{
Momento de Irrigar a Cultura de Amendoim Utilizando a Termometria a Infravermelho
}

\author{
José Eduardo Pitelli Turco $^{1}$ (D), Ana Carla Nogueira Tobias Vieira ${ }^{2}$ \\ ${ }^{1}$ Departamento de Engenharia e Ciências Exatas, Universidade Estadual Paulista \\ "Júlio de Mesquita Filho", Campus de Jaboticabal, Jaboticabal, SP, Brasil. \\ ${ }^{2}$ Programa de Pós-Graduação em Agronomia, Universidade Estadual Paulista \\ "Júlio de Mesquita Filho”, Campus de Jaboticabal, Jaboticabal, SP, Brasil.
}

Recebido em: 26 de Junho de 2020 - Aceito em: 7 de Setembro de 2020

\begin{abstract}
Resumo
O uso de índices de estresse hídrico nas fases de desenvolvimento da cultura pode atuar como uma ferramenta essencial no manejo da irrigação. Objetivou-se com este trabalho determinar o momento ideal de irrigar a cultura do amendoim, por meio de índices de estresse hídrico utilizando-se a termometria a infravermelho. A pesquisa foi desenvolvida na área experimental do Departamento de Engenharia Rural da FCAV/UNESP, Jaboticabal-SP, situada a 22 15 '22" S, 48 18 '58" W, e altitude de 575 m, em uma "Bacia Hidrográfica Experimental", utilizando superfícies com diferentes declividades $(0 \%, 20 \%$ e $40 \%)$ e exposições solares (Norte, Sul, Leste e Oeste), sob déficit hídrico induzido. O solo da área experimental é o LE1, Vermelho-Escuro, eutrófico, A moderado, caulinítico, hipoférrico, textura argilosa, relevo suave ondulado (Eutrustox). O índice de estresse hídrico foi determinado por meio da medição da temperatura da cobertura vegetal e do ar ambiente. A rega foi realizada utilizando-se sistema de irrigação por gotejamento. Os resultados indicam que o momento de irrigar difere para cada fase de desenvolvimento do amendoim, em uma faixa entre $1,3{ }^{\circ} \mathrm{C}$ até $3,3{ }^{\circ} \mathrm{C}$. A superfície mantida na capacidade de campo obteve menor índice de estresse hídrico $\left(-1,4{ }^{\circ} \mathrm{C}\right)$.
\end{abstract}

Palavras-chave: manejo de água e solo, déficit hídrico, superfícies inclinadas.

\section{The Timeto Irrigate a Peanut Crop Using Infrared Thermometry}

\begin{abstract}
The use of water stress indexes in the developmental phases of the crop can be an essential tool for irrigation control. The aim of this study was to determine the ideal time to irrigate a peanut crop, using a water stress index found using infrared thermometry. The research was developed in the experimental area of the Department of Rural Engineering of FCAV / UNESP, Jaboticabal-SP, located at $22^{\circ} 15^{\prime} 22^{\prime \prime} \mathrm{S}, 48^{\circ} 18^{\prime} 58^{\prime}$ " W, and altitude of $575 \mathrm{~m}$, in an "Hydrographic Basin" (0\%, 20\% and 40\%) and solar exposures (North, South, East and West), under induced water deficit. The soil of the experimental area is LE1, Red-Dark, eutrophic, A moderate, kaolinite, hypoferric, clayey texture, smooth undulating relief (Eutrustox). Water stress level was determined by measuring the temperatures of the vegetation cover and the ambient air. Watering was carried out using a drip irrigation system. The results indicate that the time of irrigation differs for each stage of development of the peanut, in a range between $1.3{ }^{\circ} \mathrm{C}$ and $3.3^{\circ} \mathrm{C}$. The area maintained in the field capacity obtained lower water stress index $\left(-1.4^{\circ} \mathrm{C}\right)$.
\end{abstract}

Keywords: water and soil management, water stress, sloping surfaces.

\section{Introdução}

$\mathrm{O}$ amendoim (Arachis hypogaea L.) é uma oleaginosa mundialmente cultivada, sendo o mercado mundial liderado pela China, Índia e Estados Unidos, que são, além de exportadores, os maiores consumidores.

No Brasil, a produção média é de 466 mil toneladas anuais, estando como o segundo maior produtor e exporta-

Autor de correspondência: José Eduardo Pitelli Turco, Jose.turco@unesp.br. 
dor de amendoim da América Latina, ficando atrás apenas da Argentina. O estado de São Paulo concentra mais de $90 \%$ da produção nacional de amendoim (Embrapa, 2019).

No estado de São Paulo a maior parte da produção do amendoim se dá na entressafra da produção de cana-deaçúcar, com o objetivo de promover uma renda adicional e geração de empregos durante esse período, além da melhoria da fertilidade do solo, já que se trata de uma planta leguminosa, diminuindo os custos com adubos nitrogenados no momento da implantação da cana-de-açúcar (Ambrosano et al., 2011).

No Brasil, essa oleaginosa tem sido cultivada em condições de sequeiro, sujeita a elevados riscos causados pelas variações de clima, incluindo a instabilidade de chuvas, ocasionando baixas produções. O déficit hídrico está entre os fatores que mais limitam o crescimento e a produtividade agrícola e afeta principalmente regiões sem irrigação e com má distribuição pluviométrica (Holanda et al., 2014).

Como toda planta necessita de água para crescer e se desenvolver, a irrigação pode ser viável, também, na cultura do amendoim. Em regiões onde ocorre chuva pode ter estiagens longas (veranicos), necessitado de irrigação. $\mathrm{O}$ déficit hídrico é o maior impacto negativo sobre o rendimento de cultivos de amendoim em todo o mundo (Pereira et al., 2016).

A utilização de estratégias de irrigação que venha a minimizar os impactos do déficit hídrico e melhorando o rendimento sob condições de estiagem, seria o caminho mais eficaz para o crescimento do amendoim sob fornecimento limitado de água (Thangthong et al., 2018).

Para o manejo de irrigação é necessário saber quando e quanto irrigar. Para determinar quando irrigar pode utilizar os índices de estresse hídrico, que é uma técnica indireta que conduz a determinação do momento de irrigar a cultura.

$\mathrm{Na}$ agricultura irrigada, deve-se atribuir uma atenção especial ao manejo da irrigação, a fim de garantir à disponibilidade de água durante todo o ciclo (Santos et al., 2012). Segundo Azevedo et al. (2014) a cultura deve ser irrigada sem que ocorra déficit ou excesso de água.

A temperatura do dossel é um dos melhores assimiladores da saúde das plantas e tem sido usada com sucesso para manejo da irrigação. Portanto, monitorando continuamente a temperatura do dossel da cultura usando a termometria infravermelha podem fornecer o momento de irrigar (Zonta et al., 2018).

A termometria infravermelha é um método que possibilita a medição da temperatura de uma porção do dossel vegetativo, sem a necessidade de contato físico com as folhas. E a temperatura do dossel pode ser utilizada para determinar o momento que a planta está em déficit hídrico. O índice de estresse hídrico da cultura (IEHC) que sinaliza a necessidade de irrigação é específico da cultura e deve levar em consideração fatores como a resposta ao déficit hídrico, o valor provável da cultura e custo da água (Ramírez et al., 2015).

Superfícies com diferentes exposições e declividades diferem na radiação solar acumulada, a qual é o fator que condiciona os elementos climatológicos e fisiológicos relacionados ao crescimento e estabelecimento das culturas. Informações estratégicas como topografia do terreno e temperatura do dossel vegetativo podem fornecer tecnologias de manejo acessíveis ao produtor rural (Brunini e Turco, 2016).

Levando-se em consideração os aspectos citados, objetivou-se com este trabalho determinar o momento de irrigar a cultura do amendoim, por meio de índices de estresse hídrico utilizando-se a termometria a infravermelho.

\section{Materiais e Métodos}

A pesquisa foi desenvolvida na área experimental do Departamento de Engenharia Rural da FCAV/UNESP, Câmpus de Jaboticabal-SP, situada a $22^{\circ} 15^{\prime} 22^{\prime \prime} \mathrm{S}$, $48^{\circ} 18^{\prime} 58^{\prime \prime} \mathrm{W}$, e altitude de $575 \mathrm{~m}$, em uma estrutura denominada "Bacia Hidrográfica Experimental", descrita com detalhes por Turco et al. (1997).

$\mathrm{O}$ experimento foi realizado nessa estrutura, onde foram utilizadas dez superfícies caracterizadas como $\mathrm{H} 1$ (superfície horizontal), H2 (superfície horizontal, sem ocorrência de déficit hídrico), $20 \mathrm{~N}$ (superfície com $20 \%$ de declividade e exposição norte), $40 \mathrm{~N}$ (superfície com $40 \%$ de declividade e exposição norte), $20 \mathrm{~S}$ (superfície com $20 \%$ de declividade e exposição sul), $40 \mathrm{~S}$ (superfície com $40 \%$ de declividade e exposição sul), 20E (superfície com $20 \%$ de declividade e exposição leste), 40E (superfície com $40 \%$ de declividade e exposição leste), $20 \mathrm{~W}$ (superfície com $20 \%$ de declividade e exposição oeste), $40 \mathrm{~W}$ (superfície com $40 \%$ de declividade e exposição oeste)

$\mathrm{O}$ experimento foi iniciado no dia 04 de outubro de 2017, quando foi realizada a semeadura do amendoim. Sua emergência ocorreu no dia 14 de outubro de 2017, sendo o controle da irrigação realizado do dia 24 de outubro de 2017 ao dia 23 de janeiro de 2018. No dia 03 de fevereiro de 2018 foi realizada a colheita do amendoim.

Todas as superfícies foram cultivadas com amendoim, cultivar: IAC OL3, categoria: $\mathrm{C} 2$; sendo semeadas 20 sementes por metro a $5 \mathrm{~cm}$ de profundidade.

Os dados meteorológicos referentes a todo o período de implantação e condução do experimento foram coletados por uma Estação Meteorológica Automatizada da marca Davis Instruments do Departamento de Engenharia Rural da FCAV/UNESP, Campus de Jaboticabal, abrangendo todo o ciclo da cultura do amendoim. A estação possui os seguintes sensores: temperatura e umidade relativa do ar, modelo 7859; velocidade do vento, modelo 7911; radiação solar global, modelo 6450; todos da marca Davis Instruments. 
A radiação solar global diária acumulada $\left(\mathrm{MJ} . \mathrm{m}^{-2}\right.$. $\mathrm{dia}^{-1}$ ) nas superfícies na horizontal foi obtida por dados coletados de radiação solar global da Estação Meteorológica Automatizada e estimada para outras superfícies pela metodologia utilizada por Coan et al. (2012).

A irrigação, do tipo gotejamento, em cada superfície foi realizada por meio da instalação de seis mangueiras de $3,5 \mathrm{~m}$ de comprimento, com gotejadores a cada $20 \mathrm{~cm}$, em toda a sua extensão. O conjunto possuía uma vazão de $90 \mathrm{Lh}^{-1}$.

Durante o período do desenvolvimento da cultura do amendoim (primavera e o verão), ocorrem chuvas. Quando a quantidade de chuva não era suficiente para suprir de água a lavoura foi realizada irrigação suplementar nas superfícies.

Houve três curtos períodos de deficiência hídrica, com a necessidade de irrigação suplementar, que foram utilizados para determinar o momento de irrigar a cultura do amendoim.

Nos três curtos períodos de deficiência hídrica, superfície $\mathrm{H} 2$ foi mantida na capacidade de campo, sem ocorrência de déficit hídrico induzido na condução do experimento, as outras superfícies $(\mathrm{H} 1,20 \mathrm{~N}, 40 \mathrm{~N}, 20 \mathrm{~S}$, $40 \mathrm{~S}, 20 \mathrm{E}, 40 \mathrm{E}, 20 \mathrm{~W}$, e $40 \mathrm{~W}$ ) foram irrigadas quando o armazenamento de água atingiu $50 \%$ da capacidade de água disponível do solo (Brunini e Turco, 2018).

Para a determinação o momento de irrigar as superfícies sob déficit hídrico, foi instalado no centro de cada superfície três tensiômetros (vacuômetros) a $0,20 \mathrm{~m}$ e três a $0,40 \mathrm{~m}$ de profundidade, respectivamente (região de maior concentração das raízes), centralizados em linha reta, e sentido perpendicular à declividade da superfície, com a finalidade de monitorar o comportamento do potencial matricial de água no solo (umidade crítica do solo) (Farias et al., 2009). A equação para a determinação do potencial matricial da água no solo é dada pela Eq.(1):

$$
\Psi m=-12,6 h+h_{1}+h_{2}
$$

em que $\Psi_{m}$ - potencial matricial da água no solo ( $\left.\mathrm{cmca}\right) ; h$ - altura da coluna de mercúrio $(\mathrm{cm}) ; h_{1}$ - altura da cuba de mercúrio em relação a superfície do solo $(\mathrm{cm}) ; h_{2}$ - profundidade de instalação do tensiômetro $(\mathrm{cm})$.

Para as profundidades de 0,20 e $0,40 \mathrm{~m}$ o potencial matricial da água no solo foi convertido em umidade utilizando o modelo matemático de curva de retenção de água pelo solo apresentado por Van Genuchten (1980), Eq. (2), considerando que para a umidade de capacidade de campo $(\theta c c)$ a tensão da água no solo é $103,32 \mathrm{~cm} \mathrm{H}_{2} \mathrm{O}$ e umidade atual $\left(\theta_{A}\right)$ obtida diariamente com a tensão da água a partir das leituras dos tensiômetros.

$$
\theta_{A}=\theta_{r} \frac{\left(\theta_{s}-\theta_{r}\right)}{\left(1+\left(\alpha *|\Psi m|^{n}\right)\right)^{m}}
$$

em que $\theta_{A}$ - umidade atual $\left(\mathrm{cm}^{-3} \mathrm{~cm}^{-3}\right) ; \theta_{r}$ - umidade residual $\left(\mathrm{cm}^{-3} \mathrm{~cm}^{-3}\right) ; \theta_{S}$ - umidade do solo saturado $\left(\mathrm{cm}^{-3} \mathrm{~cm}^{-3}\right) ; \Psi_{m^{-}}$potencial matricial da água no solo (cmca); $\alpha, n$ e $m$ - coeficientes de ajustes gerados pelo modelo de Van Genuchten (1980).

Na Tabela 1 é possível verificar os valores das características físico-hídricas do solo do local em que ocorreu o experimento e os valores das constantes empíricas empregadas na Eq. (2).

Também foram calculadas: a capacidade de água disponível no solo $(C A D)$, a água facilmente disponível no solo $(A F D)$ e a reserva de água disponível no solo $\left(R A D_{f}\right)$; por meio das Eqs:

$$
\begin{gathered}
C A D(m m)=\left(\theta_{C C}-\theta_{P M P}\right) * Z \\
A F D(m m)=0,5 * C A D \\
R A D_{f}(m m)=(C A D-A F D)
\end{gathered}
$$

em que $\theta_{C C}$ - umidade do solo à capacidade de campo (potencial de $103,32 \mathrm{~cm} \mathrm{H}_{2} \mathrm{O}$ ), $\mathrm{cm}^{3} \mathrm{~cm}^{-3} ; \theta_{P M P}$ - umidade do solo no ponto de murcha (potencial mátrico de $\left.15498,41 \mathrm{~cm} \mathrm{H}_{2} \mathrm{O}\right), \mathrm{cm}^{3} \mathrm{~cm}^{-3} ; Z$ - profundidade efetiva do sistema radicular do amendoim $(0,30 \mathrm{~m})$.

A quantidade de água aplicada nas superfícies foi determinada em função dos valores da ETo, obtidos pelo método de Penman-Monteith (Allen et al., 2006) e corrigido por Turco et al. (2012), com o uso do coeficiente da cultura $(K c)$ inseridos ao longo do ciclo da cultura $(0,4$; $1,15$ e 0,6$)$.

$$
E T_{c}=E T_{0} K_{c}
$$

em que $E T_{c}$ - evapotranspiração da cultura $\left(\mathrm{mm} \mathrm{d}^{-1}\right) ; E T_{o}$ evapotranspiração de referência $\left(\mathrm{mm} \mathrm{d}^{-1}\right) ; K_{c}$ - coeficiente da cultura.

Tabela 1 - Características físico-hídricas do solo do local em que ocorreu o experimento e valores das constantes empíricas empregadas na equação de Van Genuchten (1980).

\begin{tabular}{lllllllll}
\hline Profundidade $(\mathrm{m})$ & $\theta_{C C}\left(\mathrm{~cm}^{3} \mathrm{~cm}^{-3}\right)$ & $\theta_{P M P}\left(\mathrm{~cm}^{3} \mathrm{~cm}^{-3}\right)$ & $D s\left(\mathrm{~g} \mathrm{~cm}^{-3}\right)$ & $n$ & $M$ & $\alpha$ & $\theta_{S}\left(\mathrm{~cm}^{3} \mathrm{~cm}^{-3}\right)$ & $\theta_{r}\left(\mathrm{~cm}^{3} \mathrm{~cm}^{-3}\right)$ \\
\hline 0,2 & 0,3799 & 0,297 & 1,13 & 12,7127 & 0,1383 & 0,0178 & 0,539 \\
0,4 & 0,2685 & 0,209 & 1,04 & 17,7713 & 0,1432 & 0,0182 & 0,506 & 0,297 \\
\hline
\end{tabular}

$\theta c c$ - umidade do solo à capacidade de campo; $\theta_{P M P^{-}}$umidade do solo no ponto de murcha permanente; $D s$ - densidade do solo; $\theta s$ - umidade do solo saturado; $\theta r$ - umidade residual do solo; $\alpha, n$ e $m$ - coeficientes de ajuste gerados pelo modelo (Van Genuchten, 1980). 
A determinação do IEHC foi efetuada todos os dias, entre 12 e $13 \mathrm{~h}$ (horário solar), para 10 leituras em cada parcela das superfícies estudadas, por meio da medição, concomitante, da temperatura da cobertura vegetal e da temperatura do ar ambiente, com o uso do termômetro de infravermelho, portátil, FLUKE, modelo $62 \mathrm{MAX}+$ (calibrado), de ângulo de visada (ângulo sólido) de $3^{\circ}$, direcionando-se o aparelho para a cultura. Foi obtido o valor da temperatura da cobertura vegetal, para o cálculo do IEHC, pela média das dez visadas em cada parcela.

A fim de evitar erros amostrais, as leituras foram efetuadas com o sensor do aparelho posicionado em direção oposta ao sol, e o ângulo de inclinação de aproximadamente $30^{\circ}$ com a horizontal (Fernandes e Turco, 2001). Em dias de precipitações, ocorrência de vento forte (observação visual), e ou tempo nublado interceptando o fluxo de radiação solar direta, as leituras foram evitadas, de acordo com a limitação do aparelho e da metodologia.

Segundo Fernandes (2010), um valor de IEHC positivo, aponta que a cultura está sob déficit hídrico. O cálculo do $I E H C$ do amendoim foi efetuado pela diferença entre as temperaturas médias da cobertura vegetal $\left(T_{S} \mathrm{em}\right.$ $\left.{ }^{\circ} \mathrm{C}\right)$, e as temperaturas médias do ar $\left(T_{a} \mathrm{em}{ }^{\circ} \mathrm{C}\right)$, e expressa pela Eq.(7), conforme proposto por Idso et al. (1981) e Jackson et al. (1981):

$$
I E H C=T_{s}-T_{a}
$$

em que $I E H C$ - índice de estresse hídrico $\left({ }^{\circ} \mathrm{C}\right) ; T_{S}$ - temperatura média da cobertura vegetal $\left({ }^{\circ} \mathrm{C}\right) ; T_{a}$ - temperatura média do ar $\left({ }^{\circ} \mathrm{C}\right)$.

As avaliações do crescimento da cultura foram realizadas na linha central de todas as superfícies em três plantas, com três repetições, abrangendo as fases de crescimento e maturação da cultura, e suas variações no decorrer do período. Foi utilizada como bordadura a primeira linha em cada lateral e $0,50 \mathrm{~m}$ na entrada da linha analisada.

Ao final do experimento as estimativas de produtividade foram realizadas na linha central de todas as superfícies por meio do peso total por metro (vagens $9 \%$ umidade) em três repetições. Os valores foram estimados para um hectare.

$\mathrm{O}$ delineamento experimental foi inteiramente casualizado, com três repetições. Os dados foram submetidos à análise de variância pelo teste $\mathrm{F}$, e se significativo, seguido da aplicação do Teste de Tukey $(\mathrm{p}<0,05)$.

\section{Resultadose Discussão}

$\mathrm{Na}$ Tabela 2, estão apresentados os dados de precipitação ao longo do período de cultivo e as irrigações mensais, corrigidas para cada superfície, segundo Turco et al. (2012).

A precipitação total durante o ciclo de cultivo do amendoim foi de $597 \mathrm{~mm}$, o que está dentro do intervalo esperado para o clima da região. As necessidades hídricas da cultura do amendoim variam de 450 a $700 \mathrm{~mm}$ durante o ciclo. A máxima exigência hídrica ocorre durante o florescimento e frutificação (Cato et al., 2008).

Silva e Beltrão (2000) e Ferrari Neto et al. (2012) citam que o déficit hídrico pode afetar negativamente o conjunto das funções fisiológicas do amendoim, como a fotossíntese, respiração e outras reações metabólicas, que podem repercutir diretamente nas variações anatômicas (estômatos), no crescimento, na reprodução e no desenvolvimento das plantas, de modo geral, particularmente nos frutos e sementes e, conseqüentemente, na produtividade.

A Fig. 1 (A, B, C e D) apresenta os valores dos índices de estresse hídrico (IEHC) das superfícies para o amendoim em duas fases de desenvolvimento (frutificação e maturação). É possível observar que os maiores valores de IEHC foram registrados durante as fases de frutificação e maturação do amendoim durante os períodos de déficit hídrico induzido, sendo de aproximadamente $3,8^{\circ} \mathrm{C}$ para a superfície $\mathrm{H} 1 ; 3,5{ }^{\circ} \mathrm{C}$ para $20 \mathrm{~N}$ e $3,0{ }^{\circ} \mathrm{C}$ para $20 \mathrm{~S}$ (Fig. 1A). Registrou-se $3,4{ }^{\circ} \mathrm{C}$ para a superfície $40 \mathrm{~N}$ e $3,3{ }^{\circ} \mathrm{C}$ para a superfície $40 \mathrm{~S}$ (Fig. 1B). Foi registrado o valor de $2,1{ }^{\circ} \mathrm{C}$ para $20 \mathrm{E}$ e de $2,2{ }^{\circ} \mathrm{C}$ para $20 \mathrm{~W}$ (Fig. 1C). Para 40E e 40W, observaram-se valores de $3,1{ }^{\circ} \mathrm{C}$ e $3,4^{\circ} \mathrm{C}$ respectivamente (Fig. 1D). Na superfície H2 (Fig. 1A), mantida sob capacidade de campo, o maior valor registrado foi de $-1,2^{\circ} \mathrm{C}$.

Com a diminuição da transpiração foliar ocorre aumento na temperatura do dossel devido à maior concentração de energia na forma de calor sensível. Assim, a temperatura foliar torna-se superior à temperatura do ar (Brunini e Turco, 2016), explicando, assim, o comportamento do índice de estresse hídrico variar entre uma faixa de 0,1 a $3,8^{\circ} \mathrm{C}$ para as superfícies $\mathrm{H} 1,20 \mathrm{~N}, 20 \mathrm{~S}, 40 \mathrm{~N}$, 40S, 20E, 20W, 40E e 40W (Fig. 1A, B, C e D), por conta do regime hídrico adotado, da declividade e das exposições solares das superfícies, que afetam diretamente a incidência de radiação solar para cada tratamento.

De acordo com a Tabela 3, observam-se as médias dos índices de estresse hídrico para as fases de frutificação e maturação do amendoim nas superfícies estudadas, vista que para a fase de florada, não houve déficit hídrico. Para a fase de frutificação, a superfície $\mathrm{H} 1$ apresentou a maior média de índice de estresse hídrico $\left(2,7{ }^{\circ} \mathrm{C}\right)$, seguido da $20 \mathrm{~N}\left(2,2{ }^{\circ} \mathrm{C}\right)$. E na fase de maturação, dividida em duas etapas (Etapa $1=63$ a 72 DAE; Etapa $2=97$ a $102 \mathrm{DAE}$ ), a superfície $\mathrm{H} 1$ também apresentou maior índice de estresse hídrico $\left(3,3{ }^{\circ} \mathrm{C}\right.$ e $3,2{ }^{\circ} \mathrm{C}$, Etapa 1 e Etapa 2, respectivamente), seguido da $20 \mathrm{~N}\left(3,0^{\circ} \mathrm{C}\right.$ e $2,7^{\circ} \mathrm{C}$, Etapa 1 e Etapa 2, respectivamente).

A relação existente entre a necessidade hídrica das plantas e a temperatura do dossel é um parâmetro importante para indicar ao produtor quando irrigar a cultura (Nogueira e Silva Júnior, 2001). 
Com base nas médias da superfície H1 apresentadas na Tabela 3, pode-se considerar usual para o amendoim cultivado nestas condições de campo (mais comuns de plantio), a fase de maturação como a fase decisiva para fazer o correto manejo da irrigação e o momento ideal quando a temperatura do dossel atingir o valor de $3,3{ }^{\circ} \mathrm{C}$. Portanto, um valor de 14,24 $\mathrm{mm}$, dentro da Capacidade de Água Disponível do solo (CAD), que é a reserva utilizável em $\mathrm{mm}$, atingiu o valor de $50 \%$

Tabela 2 - Lâmina d'água mensal (mm) recebida pelo amendoim em cada superfície e a precipitação (mm), ocorridas durante o desenvolvimento do experimento.

\begin{tabular}{lcccccccccccc}
\hline Ano & Meses & $\begin{array}{c}\mathrm{H} 1 \\
(\mathrm{~mm})\end{array}$ & $\begin{array}{c}\mathrm{H} 2 \\
(\mathrm{~mm})\end{array}$ & $\begin{array}{c}20 \mathrm{~N} \\
(\mathrm{~mm})\end{array}$ & $\begin{array}{c}20 \mathrm{~S} \\
(\mathrm{~mm})\end{array}$ & $\begin{array}{c}40 \mathrm{~N} \\
(\mathrm{~mm})\end{array}$ & $\begin{array}{c}40 \mathrm{~S} \\
(\mathrm{~mm})\end{array}$ & $\begin{array}{c}20 \mathrm{E} \\
(\mathrm{mm})\end{array}$ & $\begin{array}{c}20 \mathrm{~W} \\
(\mathrm{~mm})\end{array}$ & $\begin{array}{c}40 \mathrm{E} \\
(\mathrm{mm})\end{array}$ & $\begin{array}{c}40 \mathrm{~W} \\
(\mathrm{~mm})\end{array}$ & $\begin{array}{c}\text { Precipitação } \\
(\mathrm{mm})\end{array}$ \\
\hline 2017 & Outubro & 8 & 9 & 8 & 8 & 8 & 7 & 8 & 7 & 8 & 8 & 56 \\
2017 & Novembro & 13 & 38 & 13 & 10 & 11 & 10 & 13 & 10 & 12 & 10 & 212 \\
2017 & Dezembro & 106 & 113 & 104 & 89 & 100 & 88 & 93 & 88 & 92 & 81 & 119 \\
2018 & Janeiro & 13 & 33 & 13 & 15 & 19 & 17 & 13 & 15 & 19 & 18 & 210 \\
Total & & 140 & 193 & 138 & 122 & 138 & 122 & 127 & 120 & 131 & 117 & 597 \\
\hline
\end{tabular}

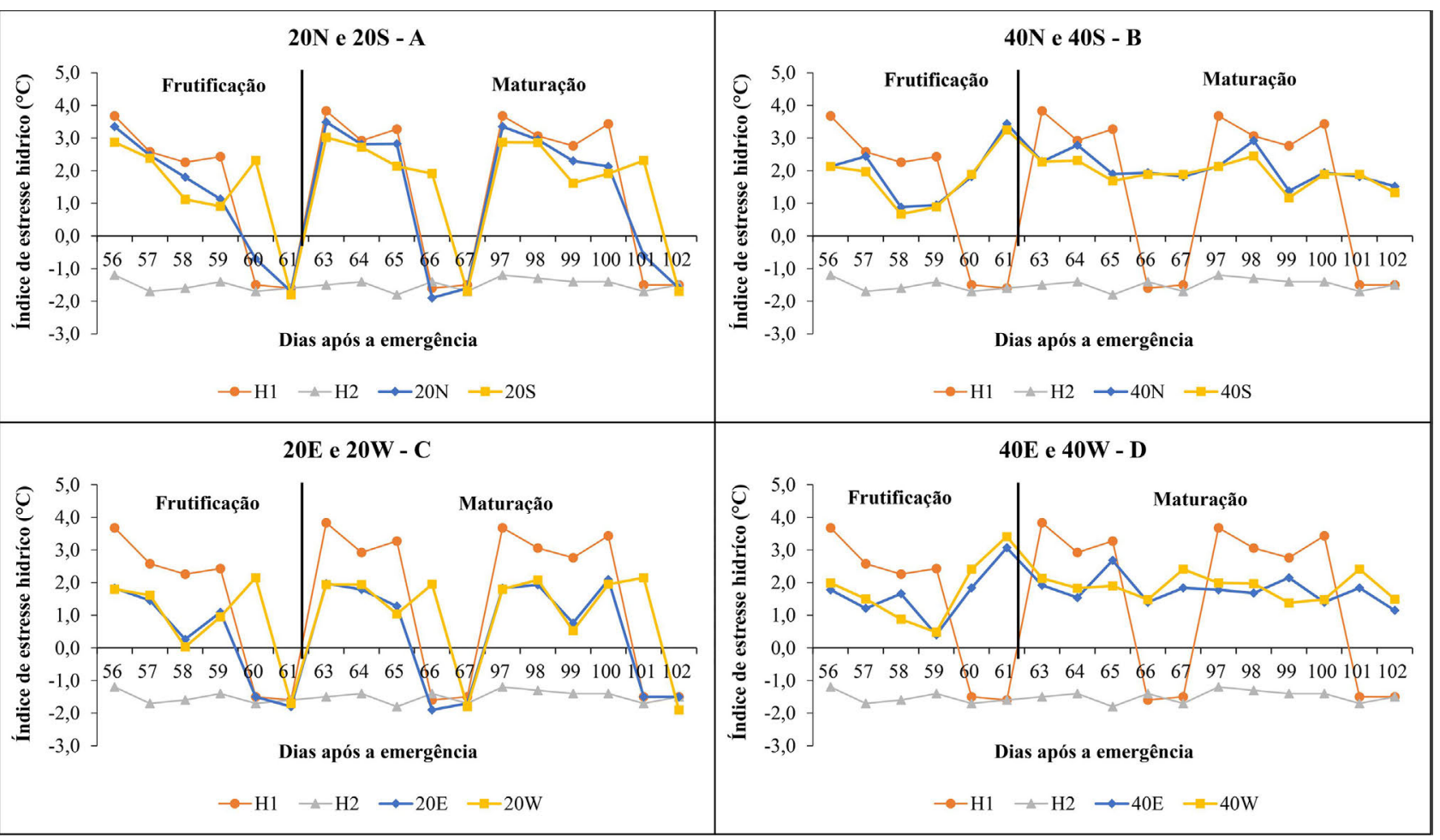

Figura 1 - Índice de estresse hídrico $\left({ }^{\circ} \mathrm{C}\right)$ para as superfícies H1, H2, 20N, 20S (A), 40N, 40S (B), 20E, 20W (C), 40E e 40W (D), no amendoim.

Tabela 3 - Análise de variância (quadrados médios) e médias dos Índices de Estresse Hídrico - IEHC $\left({ }^{\circ} \mathrm{C}\right)$ nas superfícies estudadas, para as duas fases de desenvolvimento do amendoim.

\begin{tabular}{|c|c|c|c|c|c|c|c|c|c|c|c|}
\hline \multicolumn{2}{|c|}{ IEHC } & \multirow[t]{2}{*}{$\mathrm{H} 1$} & \multirow[t]{2}{*}{$\mathrm{H} 2$} & \multirow[t]{2}{*}{$20 \mathrm{~N}$} & \multirow[t]{2}{*}{$20 \mathrm{~S}$} & \multirow[t]{2}{*}{$40 \mathrm{~N}$} & \multirow[t]{2}{*}{$40 \mathrm{~S}$} & \multirow[t]{2}{*}{$20 \mathrm{E}$} & \multirow[t]{2}{*}{$20 \mathrm{~W}$} & \multirow[t]{2}{*}{$40 \mathrm{E}$} & \multirow[t]{2}{*}{$40 \mathrm{~W}$} \\
\hline DAE & Fase & & & & & & & & & & \\
\hline 56 a 61 & Frutificação & $2,7 \mathrm{a}$ & $-1,5 \mathrm{e}$ & $2,2 \mathrm{~b}$ & $1,9 \mathrm{c}$ & $1,9 \mathrm{c}$ & $1,8 \mathrm{c}$ & $1,2 \mathrm{~d}$ & $1,3 \mathrm{~d}$ & $1,7 \mathrm{c}$ & $1,8 \mathrm{c}$ \\
\hline 63 a 67 & Maturação "” (1) & $3,3 \mathrm{a}$ & $-1,6 \mathrm{e}$ & $3,0 \mathrm{a}$ & $2,4 \mathrm{~b}$ & $2,1 \mathrm{bc}$ & $2,0 \mathrm{bc}$ & $1,7 \mathrm{c}$ & $1,7 \mathrm{c}$ & $1,9 \mathrm{c}$ & $2,0 \mathrm{bc}$ \\
\hline 97 a 102 & Maturação ${ }^{* * * *}$ (2) & $3,2 \mathrm{a}$ & $-1,4 \mathrm{e}$ & $2,7 \mathrm{~b}$ & $2,3 \mathrm{bc}$ & $2,0 \mathrm{~cd}$ & $1,8 \mathrm{~d}$ & $1,7 \mathrm{~d}$ & $1,7 \mathrm{~d}$ & $1,7 \mathrm{~d}$ & $1,8 \mathrm{~d}$ \\
\hline
\end{tabular}

$\mathrm{DAE}=$ dias após a emergência. Médias seguidas de mesmas letras, não diferem entre si, pelo Teste de Tukey $(\mathrm{P}<0,05)$.

$\mathrm{CV}=5,3 \%$.

"CV $=8,7 \%$.

$\cdots \mathrm{CV}=9,1 \%$. 
$\mathrm{Na}$ Tabela 4, verifica-se os valores de temperatura máxima da cobertura vegetal nas fases de desenvolvimento do amendoim e o acúmulo de radiação solar global para o ciclo produtivo. A superfície $\mathrm{H} 2$ registrou valores de temperatura máxima próximos de $28,0^{\circ} \mathrm{C}$ para todas as fases de desenvolvimento, comprovando a afirmativa proposta por Idso et al. (1981) e reafirmada por Brunini e Turco (2016), de que as plantas na capacidade de campo tendem a manter sua temperatura próxima a temperatura do ar e menores valores de índice de estresse hídrico. Devido a limitação hídrica, a diminuição da transpiração foliar e o aumento no acúmulo de calor sensível pela radiação solar nas folhas (Brunini e Turco, 2018), os tratamentos sob déficit hídrico $(\mathrm{H} 1,20 \mathrm{~N}, 40 \mathrm{~N}, 20 \mathrm{~S}, 40 \mathrm{~S}, 20 \mathrm{E}$, $40 \mathrm{E}, 20 \mathrm{~W}$ e $40 \mathrm{~W}$ ) apresentaram maiores variações na temperatura máxima do dossel que o tratamento $\mathrm{H} 2 \mathrm{du}$ rante o desenvolvimento do amendoim.

As temperaturas ótimas para o crescimento vegetativo do amendoim situam-se entre $25^{\circ} \mathrm{C}$ a $35^{\circ} \mathrm{C}$ (Nogueira e Távora, 2005). Temperaturas abaixo do ótimo prolongam o estádio vegetativo o que acarreta em maiores gastos com tratos culturais devido ao alongamento do ciclo da cultura (Ferrari Neto et al., 2012).

Para as superfícies horizontais, $\mathrm{H} 1$ e H2, a radiação acumulada durante o ciclo da cultura foi de $2692 \mathrm{MJ} \mathrm{m}^{-2}$ ano $^{-1}$ (Tabela 4), que foram as superfícies que tiveram maior acúmulo de radiação. A superfície $40 \mathrm{~S}$ registrou o menor acúmulo de radiação durante o ciclo, com 2489 MJ $\mathrm{m}^{-2}$ ano ${ }^{-1}$. A variação angular de incidência dos raios solares sobre as plantas diverge no saldo de radiação, desta forma diferentes cenários produtivos com dependência geográfica do terreno influenciam no saldo de radiação total pela cultura (André et al., 2010; Carneiro et al., 2013; Brunini e Turco, 2018).

A reserva utilizável de água no solo na camada monitorada de 0 a $0,4 \mathrm{~m}$ corresponde a $28,48 \mathrm{~mm}$. Ao observar os dados da Fig. 2, pode-se acompanhar o comportamento da água no solo durante os períodos de déficit hídrico induzido, que corresponde a $50 \%$ da reserva utilizável, para as superfícies durante as fases de frutificação e maturação do amendoim.

O déficit hídrico durante o desenvolvimento do amendoim prejudica vários processos fisiológicos da planta e reduz sua produtividade (Arruda et al., 2015). Os dados obtidos no trabalho desenvolvido (Tabela 5) não corroboram com que foi verificado pelos autores, o que pode ser comprovado por meio das superfícies $\mathrm{H} 1, \mathrm{H} 2$ e 20 N. H2 foi mantida durante todo o experimento em capacidade de campo e H1e $20 \mathrm{~N}$ em três períodos em déficit hídrico. Os três tratamentos apresentaram maior produtividade.

Brunini e Turco (2018) verificaram que a ausência de déficit hídrico pelo manejo da irrigação, garante um elevado rendimento agronômico. Em estudos com a canade-açúcar na mesma área experimental, os autores verificaram que o tratamento $\mathrm{H} 2$ (mantido na capacidade de

Tabela 4 - Temperatura máxima da cobertura vegetal $\left({ }^{\circ} \mathrm{C}\right)$ das superfícies $(\mathrm{H} 1, \mathrm{H} 2,20 \mathrm{~N}, 20 \mathrm{~S}, 40 \mathrm{~N}, 40 \mathrm{~S}, 20 \mathrm{E}, 20 \mathrm{~W}, 40 \mathrm{E}$ e $40 \mathrm{~W})$, nas fases de desenvolvimento do amendoim e a Radiação Solar Global incidente acumulada $\left(\mathrm{MJ} \mathrm{m}^{-2}\right.$ ano $\left.{ }^{-1}\right)$.

\begin{tabular}{lcccccccccc}
\hline Fase de desenvolvimento & $\mathrm{H} 1\left({ }^{\circ} \mathrm{C}\right)$ & $\mathrm{H} 2\left({ }^{\circ} \mathrm{C}\right)$ & $20 \mathrm{~N}\left({ }^{\circ} \mathrm{C}\right)$ & $20 \mathrm{~S}\left({ }^{\circ} \mathrm{C}\right)$ & $40 \mathrm{~N}\left({ }^{\circ} \mathrm{C}\right)$ & $40 \mathrm{~S}\left({ }^{\circ} \mathrm{C}\right)$ & $20 \mathrm{E}\left({ }^{\circ} \mathrm{C}\right)$ & $20 \mathrm{~W}\left({ }^{\circ} \mathrm{C}\right)$ & $40 \mathrm{E}\left({ }^{\circ} \mathrm{C}\right)$ & $40 \mathrm{~W}\left({ }^{\circ} \mathrm{C}\right)$ \\
\hline Frutificação & 32,7 & 27,8 & 32,1 & 31,6 & 31,3 & 31,2 & 31,1 & 31,0 & 31,0 & 31,1 \\
Maturação & 33,0 & 28,0 & 32,6 & 31,9 & 31,5 & 31,4 & 31,4 & 31,2 & 31,2 & 31,3 \\
& & \multicolumn{7}{c}{ Radiação acumulada $\left({\left.\mathrm{MJ} . \mathrm{m}^{-2} \cdot \text { ano }^{-1}\right)}\right.$} \\
Total & 2692 & 2692 & 2646 & 2634 & 2514 & 2489 & 2666 & 2630 & 2577 & 2507 \\
\hline
\end{tabular}

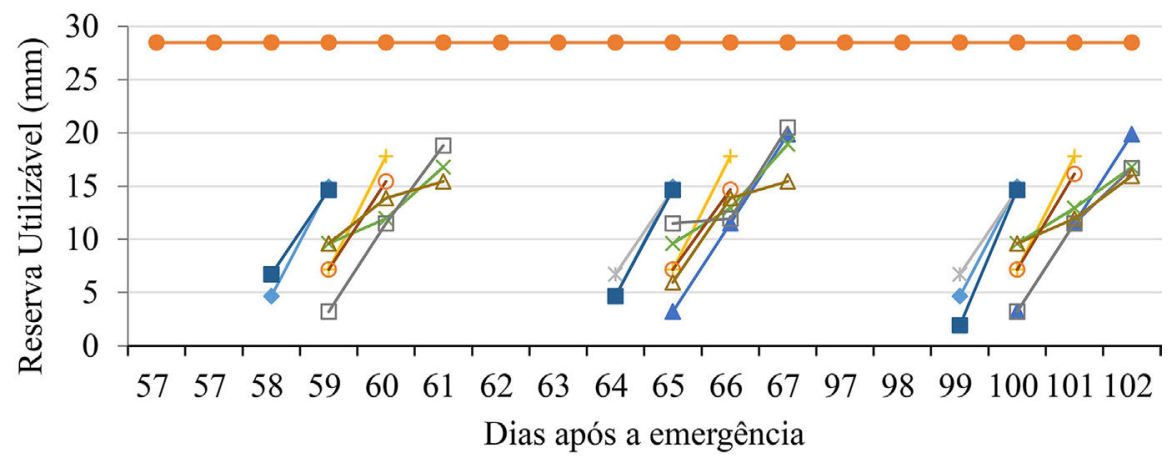

$$
\begin{aligned}
& \multimap \mathrm{H} 1-\mathrm{H} 2 \multimap-20 \mathrm{~N}-20 \mathrm{~S}-40 \mathrm{~N} \\
& \rightarrow 40 \mathrm{~S}-20 \mathrm{E}-20 \mathrm{~W} \square-40 \mathrm{E} \triangle 40 \mathrm{~W}
\end{aligned}
$$

Figura 2 - Valores médios de água disponível no solo até $0,40 \mathrm{~m}$ de profundidade, durante os períodos de déficit hídrico induzido no amendoim para as superfícies (H1, H2, 20N, 20S, 40N, 40S, 20E, 20W, 40E e 40W). 
Tabela 5 - Análise de variância (quadrados médios) e médias das produtividades do amendoim $\left(\mathrm{kg} \mathrm{ha}^{-1}\right)$.

\begin{tabular}{lcccccccccc}
\hline Superfícies & $\mathrm{H} 1$ & $\mathrm{H} 2$ & $20 \mathrm{~N}$ & $20 \mathrm{~S}$ & $40 \mathrm{~N}$ & $40 \mathrm{~S}$ & $20 \mathrm{E}$ & $20 \mathrm{~W}$ & $40 \mathrm{E}$ & $40 \mathrm{~W}$ \\
\hline Produtividade $\left(\mathrm{kg} \mathrm{ha}^{-1}\right)$ & $4159 \mathrm{a}$ & $4225 \mathrm{a}$ & $4093 \mathrm{a}$ & $3853 \mathrm{~b}$ & $3840 \mathrm{~b}$ & $3813 \mathrm{~b}$ & $3787 \mathrm{~b}$ & $3700 \mathrm{~b}$ & $3707 \mathrm{~b}$ & $2900 \mathrm{c}$
\end{tabular}

Médias seguidas de mesmas letras, não diferem entre si, pelo Teste de Tukey $(\mathrm{P}<0,05)$. CV=5,6\%.

campo) obteve maior produtividade da cultura. Os dados obtidos no trabalho desenvolvido (Tabela 5) não corroboram com dos autores, provavelmente no trabalho com cana-de-açúcar as superfícies que tiveram déficit hídrico induzido ficaram nesta condição em períodos maiores.

Conforme proposto por Idso et al. (1981), utilizar os índices de estresse hídrico na cultura do amendoim é de importância para monitorar a qualidade física e tecnológica da plantação, buscando dar suporte ao produtor rural para que ele realize o manejo da irrigação de forma correta, no momento ideal e evitando, assim, perdas na produtividade devido aos efeitos negativos que o estresse hídrico pode causar.

\section{Conclusões}

O momento de irrigar a cultura de amendoim na fase de frutificação é quando os valores médios de índices de estresse hídrico atingirem $2,7^{\circ} \mathrm{C} ; 2,2{ }^{\circ} \mathrm{C} ; 1,8{ }^{\circ} \mathrm{C}$ e $1,3{ }^{\circ} \mathrm{C}$ para as superfícies $\mathrm{H} 1 ; 20 \mathrm{~N} ; 20 \mathrm{~S} 40 \mathrm{~N}, 40 \mathrm{~S}, 40 \mathrm{E}, 40 \mathrm{~W}$; e $20 \mathrm{E}$ e $20 \mathrm{~W}$, respectivamente e na fase de maturação os valores médios de $3,2{ }^{\circ} \mathrm{C} ; 2,7^{\circ} \mathrm{C}$ e $1,8^{\circ} \mathrm{C}$ para as superfícies $\mathrm{H} 1 ; 20 \mathrm{~N}$ e $20 \mathrm{~S}, 40 \mathrm{~N}, 40 \mathrm{~S}, 20 \mathrm{E}, 20 \mathrm{~W}, 40 \mathrm{E}, 40 \mathrm{~W}$; respectivamente.

As exposições e declividades estudadas $(20 \mathrm{~N}, 40 \mathrm{~N}$, 20S, 40S, 20E, 40E, 20W e 40W) do terreno, tem influência sobre os índices de estresse hídrico para a cultura.

$\mathrm{O}$ uso da tecnologia de sensores e termômetros a infravermelho é eficaz na deteç̧ão de índice de estresse hídrico nas plantas.

\section{Agradecimentos}

Agradecemos a Coplana - Cooperativa Agroindustrial: Jaboticabal SP, pela doação da semente do amendoim.

\section{Referências}

AMBROSANO, E.J.; CANTARELLA, H.; AMBROSANO, G.M.B.; SCHAMMAS, E.A.; dias, F.L.F.; ROSSI, F.; TRIVELIN, P.C.O.; MURAOKA, T.; SACHS, R.C.C.; AZCÓN, R. Produtividade da cana-de-açúcar após o cultivo de leguminosas. Bragantia, v. 70, n. 4, p.810-818, 2011.

ALLEN, R.G.; PEREIRA, L.S.; RAES, D.; SMITH, M. Evapotraspiration Del Cultivo: Guias Para La Determinación De Los Requerimientos De Água De Los Cultivos. Roma: FAO, 298 p., 2006.

ANDRÉ, R.G.B.; MENDONÇA, J.C.; MARQUES, V.S.; PINHEIRO, F.M.A.; Marques, J. Aspectos energéticos do de- senvolvimento da cana-de-açúcar. Parte 1: balanço de radiação e parâmetros derivados. Revista Brasileira de Meteorologia, v. 25, n. 3, p. 375-382, 2010.

ARRUDA, I.M.; MODA-CIRINO, V.; BURATTO, J.S.; FERREIRA, J.M. Crescimento e produtividade de cultivares e linhagens de amendoim submetidas a déficit hídrico. Pesquisa Agropecuária Tropical, v. 45, n. 2, p. 146-154, 2015.

AZEVEDO, B.M; SOUSA G.S; PINTO PAIVA, T.F.; MESQUITA, J.B.R.; VIANA, T.V.A. Manejo da irrigação na cultura do amendoim. Magistra, v. 26, n. 1, p. 11-18, 2014.

BRUNINI, R.G., TURCO, J.E.P. Water stress indices for the sugarcane crop on different irrigated surfaces. Revista Brasileira de Engenharia Agrícola e Ambiental, v. 20, n. 10, p. 925-929, 2016.

BRUNINI, R.G.; TURCO, J.E.P. Water stress index on sugarcane in different evelopmental phases. Ciência e Agrotecnologia, v. 42, n. 2, p. 204-2015, 2018.

CARNEIRO, R.G.; MOURA, M.A.L.; LYRA, R.F.; ANDRADE, A.M.D.; SANTOS, A.B.; MAIA, R.G.X. Fluxo de calor do solo e saldo de radiação dentro de uma área de Mata Atlântica em comparação com uma área de cana-de-açúcar. Revista Brasileira de Geografia Física, v. 6, n. 3, p. 555565, 2013.

CATO, S.C.; ALBERT, L.H.B.; MONTEIRO, A.C.B.A. Amendoinzeiro. In: CASTRO, P.R.C. (eds) Manual de Fisiologia Vegetal: Fisiologia de Cultivos. Piracicaba: Editora Ceres, 2008.

COAN, R.M.; TURCO, J.E.P.; PIVETTA, K.F.L.; COSTA, M.N.; MATEUS, C.M.D’A. Emerald zoyzia grass development regarding photosynthetically active radiation in different slopes. Engenharia Agrícola, v. 32, n. 3, p. 501509, 2012.

FARIAS, C.H.A.; FERNANDES, P.D.; GHEYI, H.R.; DANTAS NETO, J. Qualidade industrial de cana-de-açúcar sob irrigação e adubação com zinco, em Tabuleiro Costeiro paraibano. Revista Brasileira de Engenharia Agrícola e Ambiental, v. 13, n. 4, p. 419-428, 2009.

EMBRAPA - Empresa Brasileira de Pesquisa Agropecuária. Estudo Mapeia Áreas De Produção De Amendoim No Brasil Para Prevenir A Doença Do Carvão. Disponível em https://www.embrapa.br/busca-de-noticias/-/noticia/ 40697528/estudo-mapeia-areas-de-producao-de-amen doim-do-brasil-para-prevenir-doenca-do-carvao. Acesso em 16/06/2019.

FERNANDES, E.J. Determinação do índice de estresse hídrico em cultura do feijoeiro com termômetro de infravermelho. Irriga, v.15, n.3, p.248, 2010.

FERNANDES, E.J.; TURCO, J.E.P. Utilização do CWSI na determinação de estresse hídrico em cultura de soja. Revista Brasileira de Agrometeorologia, v. 9, n. 2, p. $347-355,2001$. 
FERRARI NETO, J.; COSTA, C.H.M.; CASTRO, G.S.A. Ecofisiologia do amendoim. Scientia Agraria Paranaensis, v. 11, n. 4, p.1-13, 2012.

HOLANDA, L.A.D.; SANTOS, C.M.; NETO, G.D.S.; PÁDUA, S.A.; ALMEIDA, S.M. Variáveis morfológicas da cana-deaçúcar em função do regime hídrico durante o desenvolvimento inicial. Irriga, v. 19, n. 4, p. 573-584, 2014.

IDSO, S.B.; JACKSON, R.D.; PINTER JUNIOR, P.J.; REGINATO, R.J.; HATFIELD, J.L. Normalizing the stressdegree-day parameter for environmental variability. Agricultural Meteorology, v. 24, n. 1, p. 45-55, 1981.

JACKSON, R.D.; IDSO, S.B.; REGINATO, R.J.; PINTER JR.; P.J. Canopy temperature as a crop water stress indicator. Water Resour, v. 17, n.1, p.1133-1138, 1981.

NOGUEIRA, R.J.M.C.; SILVA JUNIOR, J.F. Resistência estomática, tensão de água no xilema e teor de clorofila em genótipos de gravioleira. Scientia Agrícola, v. 58, n. 3, p. 491-495, 2001.

NOGUEIRA, R.J.M.; TÁVORA, F.J.A.F. Ecofisiologia do amendoim. In: Dos Santos, R.C. (ed) O Agronegócio Do Amendoim No Brasil. Campina Grande: Embrapa Algodão, p.71-122, 2005.

PEREIRA, J.W.L;ALBUQUERQUE, M.B.;MELO FILHO, P.A; NOGUEIRA, R.J.M.C;LIMA, L.M.;SANTOS, R.C. Assessment of drought tolerance of peanut cultivars based on physiological and yield traits in a semiarid environment. Agricultural Water Management, v.166, p. 70-76, 2016.

RAMÍREZ, A.J.F.; COELHO, R.D.; PIZANI, M.A.M.; DA SILVA, C.J. Determinação do índice de estresse hídrico em tomateiros cereja (lycopersicum solanum var. cerasiforme.) com câmara infravermelha. Revista Brasileira de Agricultura Irrigada, v. 9, n. 4, p. 218-224, 2015.

SANTOS, R.C.; FREIRE, R.M.M; LIMA, L.M; ZAGONEL, G.F; COSTA, B.J. Produtividade de grãos e óleo de genóti- pos de amendoim para o mercado olequímico. Revista Ciência Agronômica, v. 43, n. 1, p. 72-77, 2012.

SILVA, L.C.; BELTRÃO, N.E.M. Incremento de fitomassa e produtividade do amendoinzeiro em função de lâmina e intervalos de irrigação. Revista Brasileira de Oleaginosas e Fibrosas, v. 4, n. 2, p. 111-121, 2000.

THANGTHONG, N.; JOGLOY, S.; JONGRUNGKLANG, N.; KVIEN, C.K.; PENSUK, V.; KESMALA, T.; VORASOOT, N. Root distribution patterns of peanut genotypes with different drought resistance levels under earlyseason drought stress. Journal of Agronomy and Crop Science, v. 204, n. 2, p. 111-122, 2018.

TURCO, J.E.; MILANI, A.P.; FERNANDES, E.J. Adequacy of the Penman-Monteith method to irrigated surfasse with diferente exposures and declivity. In: Anais International Conference of Agricultural Engineering. Valencia, 2012, CD-ROM.

TURCO, J.E.P.; PINOTTI JUNIOR, M.; FERNANDES, E.J.; RODRIGUES, T.de J.D. Adequação de um modelo de crescimento da cultura de soja para terrenos com diferentes exposições e declividades. Engenharia Agrícola, v. 17, n. 4, p.25-34, 1997.

VAN GENUCHTEN, M.T. A closed form equation for predicting the hydraulic conductivity of unsaturated soils. Soil Science Society of America Journal, v. 44, n. 1, p. 892898, 1980.

ZONTA, J.H.; MARIÇOBA, R.M.; BRANDÃO, Z.N.; CARRILLO, M.A.; BEZERRA, J.R.C. Canopy temperatures and accumulated degree days on cotton plants under water deficit. Irriga, v. 23, n. 4, p. 741-755, 2018.

License information: This is an open-access article distributed under the terms of the Creative Commons Attribution License (type CC-BY), which permits unrestricted use, distribution and reproduction in any medium, provided the original article is properly cited. 